\title{
The expression of phospholipase $A 2$ group $X$ is inversely associated with metastasis in colorectal cancer
}

\author{
MASAYA HIYOSHI $^{1}$, JOJI KITAYAMA ${ }^{1}$, SHINSUKE KAZAMA ${ }^{1}$, YOSHITAKA TAKETOMI ${ }^{2}$, \\ MAKOTO MURAKAMI ${ }^{2}$, NELSON H. TSUNO ${ }^{3}$, KUMIKO HONGO ${ }^{1}$, \\ MANABU KANEKO $^{1}$, EIJI SUNAMI ${ }^{1}$ and TOSHIAKI WATANABE ${ }^{1}$ \\ ${ }^{1}$ Division of Surgical Oncology, Department of Surgery, Faculty of Medicine, The University of Tokyo, Tokyo 113-8655; \\ ${ }^{2}$ Lipid Metabolism Project, The Tokyo Metropolitan Institute of Medical Science, Tokyo 156-8506; \\ ${ }^{3}$ Department of Transfusion Medicine, Faculty of Medicine, The University of Tokyo, Tokyo 113-8655, Japan
}

Received September 5, 2012; Accepted November 29, 2012

DOI: 10.3892/ol.2012.1067

\begin{abstract}
Among the secretory phospholipase A2s (sPLA2), sPLA2 group X (PLA2GX) has the most potent hydrolyzing activity toward phosphatidylcholine, and has recently been shown to be implicated in chronic inflammatory diseases. The aim of the present study was to investigate PLA2GX expression in colorectal cancer (CRC) and its correlation with patient clinicopathological features. The present study comprises a series of 158 patients who underwent surgical resection for primary CRC. PLA2GX expression in CRC tissues was examined by immunohistochemistry and compared with patient clinicopathological findings and survival. A total of $64 \%$ of the tumors expressed PLA2GX at high levels. Statistical analysis revealed that PLA2GX expression was inversely correlated with hematogenous metastasis $(\mathrm{P}=0.005)$. In the subgroup analysis, left-sided tumors with high PLA2GX expression showed an inverse correlation with lymph node metastasis $(\mathrm{P}=0.018)$ and hematogenous metastasis $(\mathrm{P}=0.017)$. Patients with high PLA2GX expression tended to have a longer disease-specific survival compared with those with low PLA2GX expression in left-sided, but not right-sided, $\mathrm{CRC}(\mathrm{P}=0.08)$. In light of the present results, we suggest that PLA2GX has an inhibitory effect on the progression of CRC.
\end{abstract}

\section{Introduction}

Globally, colorectal cancer (CRC) is the third most commonly diagnosed cancer in males and the second in females (1). A number of studies have demonstrated the critical involvement

Correspondence to: Dr Masaya Hiyoshi, Department of Surgery, Division of Surgical Oncology, Faculty of Medicine, The University of Tokyo, 7-3-1 Hongo, Bunkyo-ku, Tokyo 113-8655, Japan E-mail: hiyoshim-sur@h.u-tokyo.ac.jp

Key words: phospholipase A2, colorectal cancer, immunohistochemistry, metastasis of cyclooxygenase (COX) in the development and progression of CRC $(2,3)$. COX is a rate-limiting enzyme in the synthesis of bioactive prostaglandins or thromboxanes from arachidonic acid (AA), which is mainly released from membrane-bound glycerophospholipids. Phospholipase A2 (PLA2) is a key esterase that cleaves the glycerophospholipids at the sn-2 ester bond to release a fatty acid and lysophospholipid (4). Therefore, the tissue expression of PLA2 is thought to have important roles in the development of CRC.

PLA2 proteins are broadly defined into three different classes: secretory PLA2 (sPLA2), cytosolic PLA2 (cPLA2) and $\mathrm{Ca}^{2+}$-independent PLA2 (iPLA2). Approximately one-third of the PLA2s belong to the sPLA2 family, which contains typically disulfide-rich, low molecular weight enzymes with strict $\mathrm{Ca}^{2+}$ dependence and a His-Asp catalytic dyad (5). To date, 11 sPLA2s (IB, IIA, IIC, IID, IIE, IIF, III, V, X, XIIA and XIIB) have been identified in mammals. After being secreted to the extracellular space, sPLA2s act on cellular membrane-bound phospholipids in an autocrine or paracrine manner, leading to the production of various inflammatory mediators, including prostaglandins, leukotrienes and thromboxane. The other cleavage products, namely lysophospholipids, such as lysophosphatidylcholine (LPC) and lysophosphatidic acid (LPA), also have various bioactivities. Moreover, sPLA2s also act on non-cellular phospholipids, including those in microvesicles, pulmonary surfactant, lipoproteins, microbial membranes and food substances (4).

The physiological functions of the different sPLA2s have been gradually elucidated. They have been implicated in lipid digestion and obesity, activation of immune cells, asthma, atherosclerosis, acute respiratory distress syndrome and host defense against bacteria, viruses and parasites (5-8). However, differences in pathophysiological roles as well as the expression profile of each enzyme remain largely unknown. Of the sPLA2 family, sPLA2 group X (PLA2GX) has the most powerful AA-releasing activity from cell membrane-bound phospholipids, leading to eicosanoid formation $(9,10)$. Morioka et al have shown that PLA2GX also releases AA from cultured human colon carcinoma cell lines, leading to COX-2dependent PGE2 formation (11). The authors also showed 
enhanced expression of PLA2GX in adenocarcinoma cells in comparison with the normal colonic epithelia, by immunohistochemistry. PLA2GX has also been shown to stimulate the proliferation of colon cancer cells (12). From these data, the positive role of PLA2GX on colorectal carcinogenesis is speculated. In fact, previous studies have also described the expression of PLA2GX in human colon cancer tissue at the mRNA (13) and protein (14) levels. However, the precise expression and distribution patterns of PLA2GX in colonic cancer tissues remain to be characterized. In the present study, we aimed to examine the expression of PLA2GX in human $\mathrm{CRC}$ tissue and its possible correlation with clinical and pathological variables as well as with patient outcome.

\section{Patients and methods}

Patients and samples. A total of 158 consecutive patients with colorectal adenocarcinoma who underwent curative resection with lymph node dissection at the University of Tokyo Hospital (Tokyo, Japan), in the period between January 1991 and March 1994, were enrolled. There were 96 males and 62 females (mean age, 62 years; range, 38-90 years). Cases of ulcerative colitis and familial adenomatous polyposis were excluded from this study. None of the patients had received preoperative chemotherapy or radiation therapy. All pertinent clinical and histopathological data of the patients and their tumors were collected from the patients' case records. Clinicopathological features were analyzed based on the TNM classification of malignant tumors of the Union for International Cancer Control (UICC; 7th edition). All patients had been subsequently followed up at regular clinical visits until mortality or when last seen alive, for a mean observation period of 108 months. Informed consent was obtained from all patients and the study was approved by the Ethics Committee of the Hospital of the University of Tokyo, Tokyo, Japan.

The surgically resected specimens were immediately fixed in $10 \%$ buffered formalin and the cross-sections of the entire cancerous lesion were embedded in paraffin. Conventional pathological diagnosis of the primary lesion and the dissected lymph nodes was performed on hematoxylin and eosin (H\&E)stained sections. PLA2GX expression in the cancerous lesion was examined by immunohistochemical staining, as described below.

Immunohistochemical study. Rabbit anti-sPLA2GX polyclonal antibody was generated by the immunization of rabbit with a polypeptide at The Tokyo Metropolitan Institute of Medical Science (Tokyo, Japan). The specificity and immunoreactivity of the antibody was verified by immunoblotting with sPLA2-transfected cells (15). Consecutive formalin-fixed paraffin-embedded sections ( $4 \mu \mathrm{m}$ thick) were immunohistochemically stained by the streptavidin-biotin (SAB) immunoperoxidase method. For immunohistochemical staining, the sections were deparaffinized with xylene and dehydrated with $98 \%$ ethanol, placed in $0.01 \mathrm{M}$ sodium citrate buffer $(\mathrm{pH} \mathrm{6.0)}$ and heated in an autoclave oven for $15 \mathrm{~min}$. After washing twice in PBS, endogenous peroxidase activity was inhibited by incubation with $0.3 \%$ hydrogen peroxide in methanol for $20 \mathrm{~min}$. After three washes in PBS, non-specific reactions were blocked by incubation with $10 \%$ goat serum for $30 \mathrm{~min}$ at room temperature. Biotinylated goat anti-rabbit immunoglobulin and SAB complex, supplied commercially [Histfine SAB-PO(R) kit, Nichirei, Tokyo, Japan] were used as the reagents in the subsequent steps. The sections were incubated with the anti-PLA2GX antibody overnight at $4^{\circ} \mathrm{C}$. The color was then developed with diaminobenzidine solution. The sections were then lightly counterstained with a cocktail of Mayer's/Lillie-Mayer's hematoxylin and mounted. Spermatozoa were used as a positive control (16). For the negative control, the antibody was replaced with PBS.

Evaluation of immunostaining. The expression of PLA2GX in the cancerous lesion and in the surrounding normal mucosa was assessed by two observers (S.K. and M.H.) without knowledge of the corresponding clinical data. All tissue samples were assessed in a consecutive analysis to ensure maximal internal consistency. For the objective assessment of the PLA2GX expression level, it was stratified into three groups, as follows: -, not detected; + , focally positive in cancer cells; ++ , diffusely positive in carcinoma cells. The consistency between the observers was $80.1 \%$ ( $\kappa$ test), and in the discrepant cases, a consensus was reached after a joint review. In the statistical analysis, - and + were considered to be the low expression group, and ++ was considered to be the high expression group.

Statistical analysis. The statistical significance of the differences was evaluated by $\chi^{2}$ test, Fisher's exact test or a non-paired Student's t-test, as appropriate. The disease-specific survival (DSS) rate was analyzed by the Kaplan-Meier method and the log-rank comparison test. To assess the value of PLA2GX as an independent predictor, a multivariate survival analysis was performed, using the Cox proportional hazards regression model. All statistical analyses were performed with JMP 9.0 (SAS Institute, Cary, NJ, USA). P $<0.05$ was considered to indicate a statistically significant result.

\section{Results}

PLA2GX expression in human CRC. The staining patterns of PLA2GX in CRC specimens are shown in Fig. 1. In the normal colonic mucosa adjacent to the CRC, most of the colonic epithelial cells showed a weak expression of PLA2GX (Fig. 1A), although there was a variation of immunoreactivity among the cases. In the majority of the 158 tumors, PLA2GX expression was found predominantly in the cytoplasm of carcinoma cells and, compared with the normal epithelium, the staining signal was generally enhanced (Fig. 1B). The stromal tissue was not stained in any analyzed specimen. In 101 cases, PLA2GX expression was diffusely and almost equally detected in most of the cancer cells, as shown in Fig. 1B, whereas in 54 cases, the expression was observed only in focal cancer cells (Fig. 1D). In 3 cases, however, negligible staining of cancer cells was found. In addition, PLA2GX was hardly detected in the hepatic metastatic lesions, although the expression was diffuse in the primary lesion. (Fig. 1E and F). For further analyses, the tumors were divided into high expression (101 cases) and low expression (57 cases) groups. 

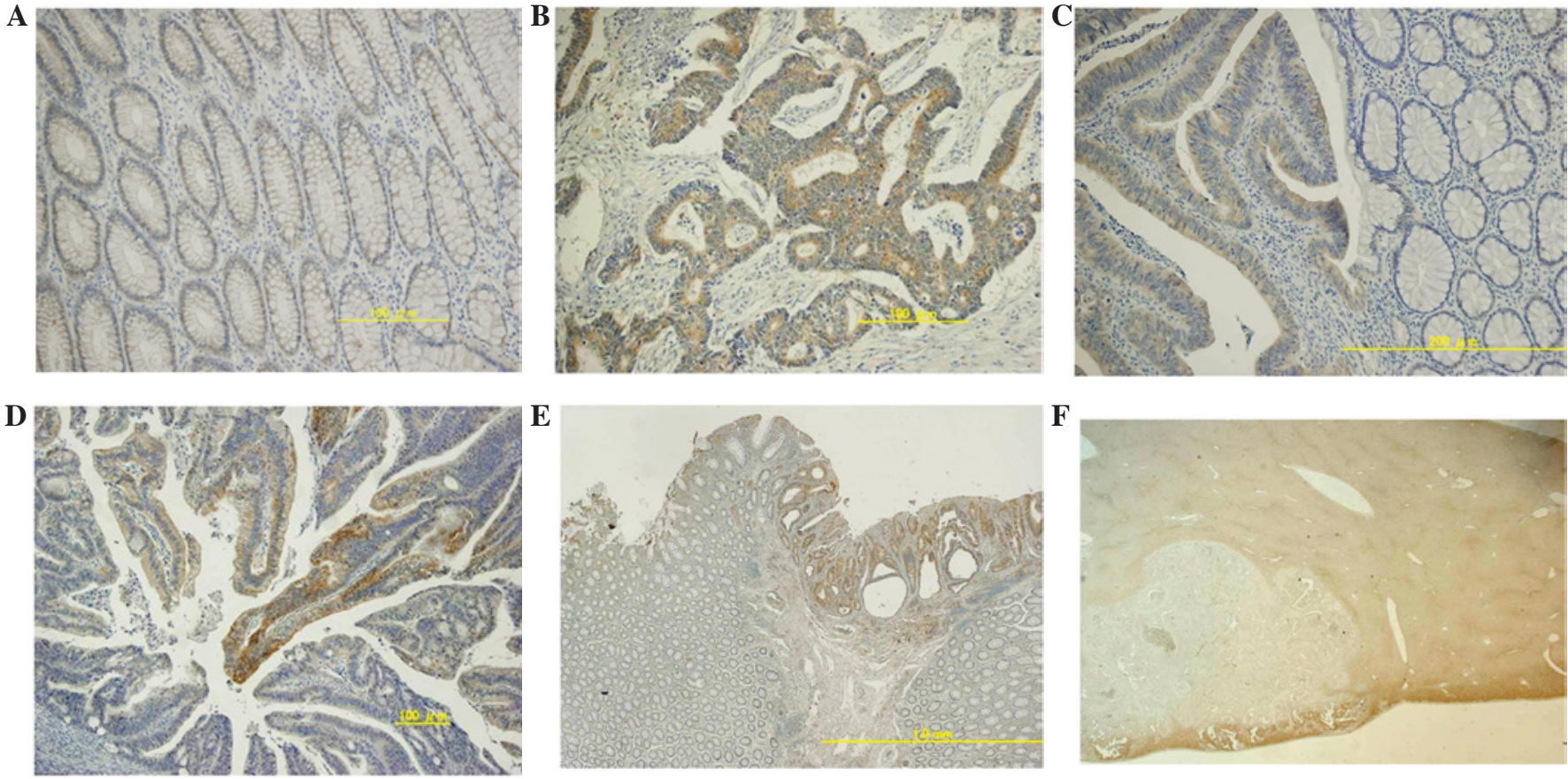

Figure 1. Expression pattern of PLA2GX in CRC specimens and normal counterparts. Staining in (A) the normal colonic mucosa (weak staining); (B) the cancer tissue (diffuse staining); (C) the boundary area between normal (right) and cancer (left); and (D) the cancer tissue (focal immunoreactivity). Representative cases of (E) primary CRC and (F) hepatic metastasis. PLA2GX, secretory phospholipase A2 group X; CRC, colorectal cancer. (A and B) Magnification, x200. (C-E) Magnification, x100. (F) Magnification, x20.
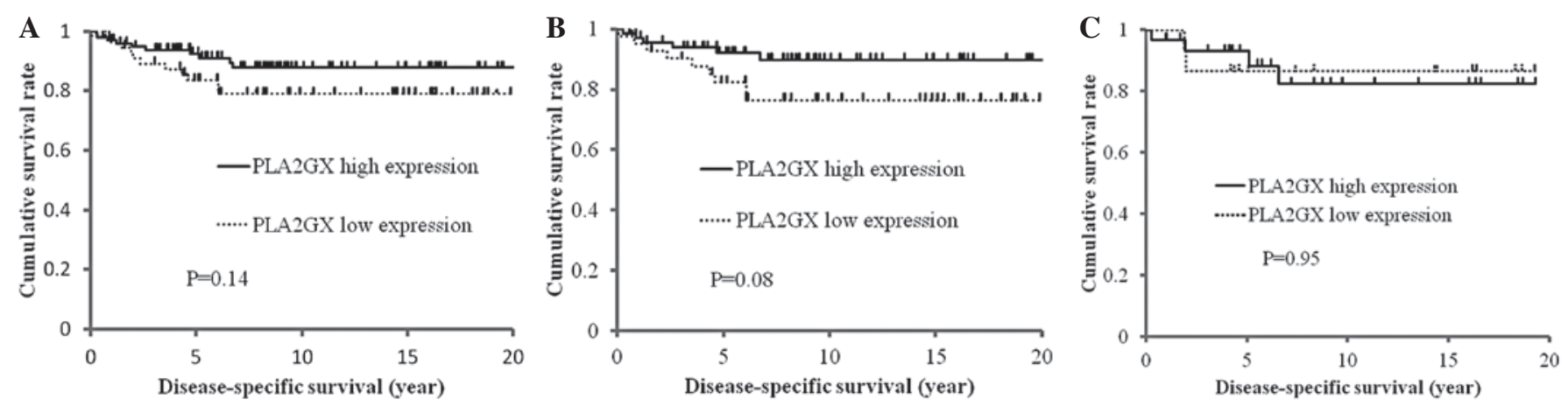

Figure 2. Survival outcomes of the patients grouped according to the expression of PLA2GX. Kaplan-Meier estimates of disease-specific survival of (A) all CRC patients, and those of (B) left- and (C) right-sided colon cancer. PLA2GX, phospholipase A2 group X.

Correlation between PLA2GX expression and clinicopathological features. Descriptive characteristics of the study subjects are presented in Table I. The expression of PLA2GX showed no correlation with age, gender, tumor size, tumor location, histological appearance, lymphatic invasion or venous involvement. However, the rate of hematogenous metastasis was significantly higher in the low PLA2GX expression group $(10.5 \%)$ than in the high PLA2GX expression group (1.0\%; $\mathrm{P}=0.005)$. Similarly, there was a tendency for higher incidence of lymph node metastasis in the low PLA2GX expression group $(50.9 \%)$ than in the high expression group $(36.6 \%$; $\mathrm{P}=0.081$ ) (Table II). When the study sample was restricted to left-sided tumors (112 cases), nodal metastasis was observed in $52.4 \%$ (22/42) of the low PLA2GX expression group, which was significantly higher than that in the high expression group $(30.0 \% ; 21 / 70 ; \mathrm{P}=0.018)$. By contrast, when samples were restricted to right-sided tumors, no difference in the incidence of nodal metastasis was observed. Additionally, only border- line significance $(\mathrm{P}=0.051)$ was observed in the association between the PLA2GX expression and the UICC stage.

Overall survival and DSS analysis of CRC with regard to PLA2GX expression. Next, we examined the correlation between PLA2GX expression and the outcome of patients, by the Kaplan-Meier analysis and the log-rank test. As shown in Fig. 2A, the high PLA2GX expression group tended to have a longer DSS, although the difference did not reach statistical significance $(\mathrm{P}=0.14)$. This trend was pronounced in left-sided $\mathrm{CRC}(\mathrm{P}=0.08)$ and was observed in right-sided CRC (Fig. 2B).

\section{Discussion}

Due to their functional diversity, PLA2 enzymes have been implicated in various biological processes, including arthritis, asthma, defense against microbes, digestion, atherosclerosis 
Table I. Association of PLA2GX expression with clinical variables.

\begin{tabular}{|c|c|c|c|c|}
\hline \multirow[b]{2}{*}{ Factor } & \multirow[b]{2}{*}{$\mathrm{n}$} & \multicolumn{2}{|c|}{ PLA2GX } & \multirow[b]{2}{*}{ P-value } \\
\hline & & High expression $(n=101)$ & Low expression $(\mathrm{n}=57)$ & \\
\hline Age (years), mean \pm SD & 158 & $62.4 \pm 11.1$ & $63.6 \pm 10.3$ & 0.481 \\
\hline \multicolumn{5}{|l|}{ Gender, n $(\%)$} \\
\hline Male & 96 & $41(40.6)$ & $21(36.8)$ & \multirow[t]{2}{*}{0.642} \\
\hline Female & 62 & $60(59.4)$ & $36(63.2)$ & \\
\hline Size of tumor $(\mathrm{mm})$, mean $\pm \mathrm{SD}$ & 158 & $46.5 \pm 24.1$ & $47.3 \pm 17.7$ & 0.824 \\
\hline \multicolumn{5}{|l|}{ T stage ${ }^{\mathrm{a}}, \mathrm{n}(\%)$} \\
\hline $\mathrm{T} 1 / \mathrm{T} 2$ & 36 & $27(26.7)$ & $9(15.8)$ & \multirow[t]{2}{*}{0.108} \\
\hline $\mathrm{T} 3 / \mathrm{T} 4$ & 122 & $74(73.3)$ & $48(84.2)$ & \\
\hline \multicolumn{5}{|l|}{ Histological type, n (\%) } \\
\hline Well, mod differentiated & 152 & $96(95.0)$ & $56(98.2)$ & \multirow[t]{2}{*}{0.285} \\
\hline Muc, por differentiated & 6 & $5(5.0)$ & $1(1.8)$ & \\
\hline \multicolumn{5}{|l|}{ Lymphatic invasion, n (\%) } \\
\hline Absent & 118 & $76(75.2)$ & $42(73.7)$ & \multirow[t]{2}{*}{0.829} \\
\hline Present & 40 & $25(24.8)$ & $15(26.3)$ & \\
\hline \multicolumn{5}{|l|}{ Lymph node metastasis, $\mathrm{n}(\%)$} \\
\hline Absent & 92 & $64(63.4)$ & $28(49.1)$ & \multirow[t]{2}{*}{0.081} \\
\hline Present & 66 & $37(36.6)$ & $29(50.9)$ & \\
\hline \multicolumn{5}{|l|}{ Venous involvement, $\mathrm{n}(\%)$} \\
\hline Absent & 75 & $50(49.5)$ & 25 (43.9) & \multirow[t]{2}{*}{0.495} \\
\hline Present & 83 & $51(50.5)$ & $32(56.1)$ & \\
\hline \multicolumn{5}{|l|}{ Location of the tumor, $\mathrm{n}(\%)$} \\
\hline Colon & 117 & $78(77.2)$ & $39(68.4)$ & \multirow[t]{2}{*}{0.229} \\
\hline Rectum & 41 & $23(22.8)$ & 18 (31.6) & \\
\hline Right side & 46 & $31(30.7)$ & $15(26.3)$ & \multirow[t]{2}{*}{0.559} \\
\hline Left side & 112 & $70(69.3)$ & $42(73.7)$ & \\
\hline \multicolumn{5}{|l|}{ UICC stage, $\mathrm{n}(\%)$} \\
\hline $\mathrm{I} / \mathrm{II}$ & 91 & $64(63.4)$ & $27(47.4)$ & \multirow[t]{2}{*}{0.051} \\
\hline III/IV & 67 & $37(36.6)$ & $30(52.6)$ & \\
\hline \multicolumn{5}{|l|}{ Hematogenous metastasis, $\mathrm{n}(\%)$} \\
\hline Absent & 151 & $100(99.0)$ & $51(89.5)$ & \multirow[t]{2}{*}{0.005} \\
\hline Present & 7 & $1(1.0)$ & $6(10.5)$ & \\
\hline
\end{tabular}

UICC, Union for International Cancer Control; ${ }^{\mathrm{T} T M N}$ classification of malignant tumors, 7th edition according to UICC. PLA2GX, secretory phospholipase A2 group X; muc, mod, mucinous; moderately; por, poorly.

and cancer (17). PLA2GX is known as the most potent sPLA2 capable of hydrolyzing phosphatidylcholine and acting extracellularly on cellular membranes and noncellular phospholipid substrates (5,9-11). It has been confirmed that PLA2GX, as well as other sPLA2s, including GIIA, GIII and GXIIA, are highly expressed in CRC tissue $(13,14,18)$. However, no information is available concerning the correlation between PLA2GX expression in cancer and clinicopathological features. Thus, in the present study, we aimed to investigate the expression of PLA2GX in patients with CRC at various stages.

In our series, the majority of the carcinoma cells in primary CRC showed enhanced cytoplasmic expression of
PLA2GX as compared with normal colonic epithelia, which is consistent with previous results $(11,13,14)$. However, in $64 \%$ of the 158 cases, PLA2GX expression was diffusely detected, while in certain cases PLA2GX was only partially expressed and in a few cases, its expression was negative. In these cases, a significant inverse correlation was found between PLA2GX expression and hematogenous metastasis and, although without significance, also with nodal metastasis. As a consequence, patients with tumors with high PLA2GX expression had a better outcome than those with low expression. To the best of our knowledge, this is the first study to show the inverse correlation of the expression of PLA2GX 
Table II. Association of PLA2GX expression in the left side of the colon with clinical variables.

\begin{tabular}{|c|c|c|c|c|}
\hline \multirow[b]{2}{*}{ Factor } & \multirow[b]{2}{*}{$\mathrm{n}$} & \multicolumn{2}{|c|}{ PLA2GX, n (\%) } & \multirow[b]{2}{*}{ P-value } \\
\hline & & High expression $(n=70)$ & Low expression $(n=42)$ & \\
\hline \multicolumn{5}{|c|}{ Lymphatic invasion } \\
\hline Absent & 85 & $54(77.1)$ & $31(73.8)$ & 0.691 \\
\hline Present & 27 & $16(22.9)$ & $11(26.2)$ & \\
\hline \multicolumn{5}{|c|}{ Lymph node metastasis } \\
\hline Absent & 69 & $49(70.0)$ & $20(47.6)$ & 0.018 \\
\hline Present & 43 & $21(30.0)$ & $22(52.4)$ & \\
\hline \multicolumn{5}{|c|}{ Venous involvement } \\
\hline Absent & 54 & $35(50.0)$ & $19(45.2)$ & 0.625 \\
\hline Present & 58 & $35(50.0)$ & $23(54.8)$ & \\
\hline \multicolumn{5}{|c|}{ Hematogenous metastasis } \\
\hline Absent & 105 & 69 (98.6) & $36(85.7)$ & 0.017 \\
\hline Present & 7 & $1(1.4)$ & $6(14.3)$ & \\
\hline
\end{tabular}

PLA2GX, secretory phospholipase A2 group X.

with the outcome of CRC patients, and the possibility that this enzyme has suppressive effects on tumor metastasis in CRC is suggested.

Increased expression of sPLA2 has been demonstrated in numerous types of cancer, including breast $(19,20)$, pancreatic $(21)$, prostate $(22,23)$, liver $(24)$, gastric $(25,26)$ and colorectal $(14,27)$ cancer. Of these, group IIA PLA2 (PLA2GIIA) is one of the isoforms most commonly investigated in cancer tissue. Kashiwagi et al reported that the high expression of PLA2GIIA was correlated with longer survival in pancreatic cancer (28). More recently, Xing et al have shown the same tendency in gastric cancer, and suggested that PLA2GIIA may negatively affect the metastatic potential of gastric cancer cells (26). In fact, Ganesan et al have demonstrated that the silencing of the PLA2GIIA gene enhances the invasive activity of tumor cells, whereas enforced expression inhibited invasion (29). These results are consistent with our finding on PLA2GX, suggesting a possibility that PLA2GX, as well as PLA2GIIA, may play negative roles in the metastatic potential of gastrointestinal cancer. However, Buhmeida et al have shown a negative association of the expression of PLA2GIIA with the prognosis of stage II CRC (27). Also, Graff et al have shown that PLA2GIIA expression increases with the progression of prostate cancer in an androgen-independent manner (30). These controversial results suggest that the role of PLA2GIIA is dependent on the interaction of cancer cells with the microenvironment in different tissues.

Surrel et al have reported that, in vitro, the addition of PLA2GX stimulates the proliferative ability of colon cancer cells by producing various lipid mediators (12). However, in their study, the proliferation of the cells was examined in serum-free media, and the effect was not completely abrogated by COX and lipooxygenase inhibitors, suggesting the involvement of other lipid mediators. The released AA itself induces the apoptosis of diverse cells, including human colon cancer cells, via an intracellular ceramide-mediated pathway (31). Ceramide acts as a second messenger in the activation process of the cellular apoptotic machinery (31). Diets rich in unsaturated fatty acids such as AA are associated with a decreased incidence of colon cancer (32). On the bases of these facts, the negative effect of PLA2GX may be attributed to the increased levels of AA produced and the subsequent increased susceptibility to apoptosis.

Although there is no significant difference between PLA2GX expression and the tumor location, the rate of lymph node metastasis was found to be significantly lower and the DSS better in left-sided CRC than in right-sided. This finding suggests the possibility that CRCs developing in the two different colonic sites may have different biological behaviors. It is known that right- and left-sided colonic tumors have different molecular profiles, with microsatellite instability and methylator phenotypes being prevalent in right-sided tumors and chromosomal instability being predominant in left-sided tumors, resulting in different biological features $(33,34)$. It is possible that these molecular profiles are intimately associated with the different lymphatic metastatic potentials. Indeed, the PLA2GX gene expression level was reported to be significantly higher in the normal mucosa of the left side of the colon than in that of the right side (13).

In conclusion, our study showed an inverse association between the reduced expression of PLA2GX and the increased metastatic potential of human CRC, especially in the left-sided tumors. Our data suggest that PLA2GX may have a protective role against the invasive ability of CRC, and the reduced ability to produce PLA2GX may result in the acquisition of a clinically more malignant phenotype. Thus, PLA2GX expression may be a potential clinical biomarker for the prediction of the invasive ability of human CRC. To date, several inhibitors of PLA2s have been developed as anticancer drugs (35). However, since PLA2s, in certain situations, may also have a suppressive effect on tumor progression, they may 
be two-edged swords, and thus their indication should be carefully considered.

\section{References}

1. Jemal A, Bray F, Center MM, Ferlay J, Ward E and Forman D: Global cancer statistics. CA Cancer J Clin 61: 69-90, 2011.

2. Williams CS, Tsujii M, Reese J, Dey SK and DuBois RN: Host cyclooxygenase-2 modulates carcinoma growth. J Clin Invest 105: 1589-1594, 2000.

3. Rodrigues S, Bruyneel E, Rodrigue CM, Shahin E and Gespach C: Cyclooxygenase 2 and carcinogenesis. Bull Cancer 91 (Suppl 2): S61-S76, 2004 (In French).

4. Murakami M, Taketomi Y, Sato H and Yamamoto K: Secreted phospholipase A2 revisited. J Biochem 150: 233-255, 2011.

5. Lambeau G and Gelb MH: Biochemistry and physiology of mammalian secreted phospholipases A2. Annu Rev Biochem 77: 495-520, 2008

6. Touqui $\mathrm{L}$ and $\mathrm{Wu} \mathrm{YZ}$ : Interaction of secreted phospholipase A2 and pulmonary surfactant and its pathophysiological relevance in acute respiratory distress syndrome. Acta Pharmacol Sin 24: 1292-1296, 2003.

7. Triggiani M, Granata F, Frattini A and Marone G: Activation of human inflammatory cells by secreted phospholipases A2. Biochim Biophys Acta 1761: 1289-1300, 2006.

8. Nevalainen TJ, Graham GG and Scott KF: Antibacterial actions of secreted phospholipases A2. Review. Biochim Biophys Acta 1781: 1-9, 2008.

9. Hanasaki K, Ono T, Saiga A, et al: Purified group X secretory phospholipase $\mathrm{A}(2)$ induced prominent release of arachidonic acid from human myeloid leukemia cells. J Biol Chem 274: 34203-34211, 1999.

10. Murakami M, Kambe T, Shimbara S, et al: Different functional aspects of the group II subfamily (Types IIA and V) and type $\mathrm{X}$ secretory phospholipase $\mathrm{A}(2) \mathrm{s}$ in regulating arachidonic acid release and prostaglandin generation. Implications of cyclooxygenase- 2 induction and phospholipid scramblase-mediated cellular membrane perturbation. J Biol Chem 274: 31435-31444, 1999.

11. Morioka Y, Ikeda M, Saiga A, et al: Potential role of group X secretory phospholipase $\mathrm{A}(2)$ in cyclooxygenase-2-dependent PGE(2) formation during colon tumorigenesis. FEBS Lett 487: 262-266, 2000

12. Surrel F, Jemel I, Boilard E, et al: Group X phospholipase A2 stimulates the proliferation of colon cancer cells by producing various lipid mediators. Mol Pharmacol 76: 778-790, 2009.

13. Mounier CM, Wendum D, Greenspan E, Fléjou JF, Rosenberg DW and Lambeau G: Distinct expression pattern of the full set of secreted phospholipases A2 in human colorectal adenocarcinomas: sPLA2-III as a biomarker candidate. Br J Cancer 98: 587-595, 2008

14. Tribler L, Jensen LT, Jørgensen K, et al: Increased expression and activity of group IIA and X secretory phospholipase A2 in peritumoral versus central colon carcinoma tissue. Anticancer Res 27: 3179-3185, 2007.

15. Degousee N, Ghomashchi F, Stefanski E, et al: Groups IV, V, and $\mathrm{X}$ phospholipases A2s in human neutrophils: role in eicosanoid production and gram-negative bacterial phospholipid hydrolysis. J Biol Chem 277: 5061-5073, 2002.

16. Masuda S, Murakami M, Matsumoto S, et al: Localization of various secretory phospholipase A2 enzymes in male reproductive organs. Biochim Biophys Acta 1686: 61-76, 2004.

17. Murakami M, Taketomi Y, Miki Y, Sato H, Hirabayashi T and Yamamoto K: Recent progress in phospholipase $\mathrm{A}_{2}$ research: from cells to animals to humans. Prog Lipid Res 50: 152-192, 2011.
18. Murakami M, Masuda S, Shimbara S, Ishikawa Y, Ishii T and Kudo I: Cellular distribution, post-translational modification, and tumorigenic potential of human group III secreted phospholipase A(2). J Biol Chem 280: 24987-24998, 2005.

19. Yamashita S, Yamashita J, Sakamoto K, et al: Increased expression of membrane-associated phospholipase A2 shows malignant potential of human breast cancer cells. Cancer 71: 3058-3064, 1993

20. Yamashita S, Yamashita J and Ogawa M: Overexpression of group II phospholipase A2 in human breast cancer tissues is closely associated with their malignant potency. Br J Cancer 69: 1166-1170, 1994.

21. Kiyohara H, Egami H, Kako H, et al: Immunohistochemical localization of group II phospholipase A2 in human pancreatic carcinomas. Int J Pancreatol 13: 49-57, 1993.

22. Jiang J, Neubauer BL, Graff JR, et al: Expression of group IIA secretory phospholipase A2 is elevated in prostatic intraepithelial neoplasia and adenocarcinoma. Am J Pathol 160: 667-671, 2002.

23. Dong Q, Patel M, Scott KF, Graham GG, Russell PJ and Sved P: Oncogenic action of phospholipase A2 in prostate cancer. Cancer Lett 240: 9-16, 2006.

24. Ying Z, Tojo H, Komatsubara T, et al: Enhanced expression of group II phospholipase A2 in human hepatocellular carcinoma. Biochim Biophys Acta 1226: 201-205, 1994.

25. Leung SY, Chen X, Chu KM, et al: Phospholipase A2 group IIA expression in gastric adenocarcinoma is associated with prolonged survival and less frequent metastasis. Proc Natl Acad Sci USA 99: 16203-16208, 2002

26. Xing XF, Li H, Zhong XY, et al: Phospholipase A2 group IIA expression correlates with prolonged survival in gastric cancer. Histopathology 59: 198-206, 2011.

27. Buhmeida A, Bendardaf R, Hilska M, et al: PLA2 (group IIA phospholipase A2) as a prognostic determinant in stage II colorectal carcinoma. Ann Oncol 20: 1230-1235, 2009.

28. Kashiwagi M, Friess H, Uhl W, et al: Group II and IV phospholipase $\mathrm{A}(2)$ are produced in human pancreatic cancer cells and influence prognosis. Gut 45: 605-612, 1999.

29. Ganesan K, Ivanova T, Wu Y, et al: Inhibition of gastric cancer invasion and metastasis by PLA2G2A, a novel beta-catenin/TCF target gene. Cancer Res 68: 4277-4286, 2008.

30. Graff JR, Konicek BW, Deddens JA, et al: Expression of group IIa secretory phospholipase A2 increases with prostate tumor grade. Clin Cancer Res 7: 3857-3861, 2001.

31. Chan TA, Morin PJ, Vogelstein B and Kinzler KW: Mechanisms underlying nonsteroidal antiinflammatory drug-mediated apoptosis. Proc Natl Acad Sci USA 95: 681-686, 1998

32. Reddy BS: Chemoprevention of colon cancer by dietary fatty acids. Cancer Metastasis Rev 13: 285-302, 1994.

33. Bendardaf R, Lamlum H, Ristämaki R, Korkeila E, Syrjänen K and Pyrhönen S: Mismatch repair status is a predictive factor of tumour response to 5-fluorouracil and irinotecan chemotherapy in patients with advanced colorectal cancer. Tumour Biol 28: 212-220, 2007.

34. Bendardaf R, Lamlum H, Ristamäki R, Korkeila E, Syrjänen K and Pyrhönen S: Thymidylate synthase and microsatellite instability in colorectal cancer: implications for disease free survival, treatment response and survival with metastases. Acta Oncol 47: 1046-1053, 2008.

35. Cummings BS: Phospholipase A2 as targets for anti-cancer drugs. Biochem Pharmacol 74: 949-959, 2007. 